\title{
SUPPORT FOR B2B E-CONTRACTING - THE PROCESS PERSPECTIVE
}

\author{
Samuil Angelov, Paul Grefen \\ Computer Science Department \\ University of Twente \\ The Netherlands \\ sangelov@cs.utwente.nl \\ grefen@cs.utwente.nl
}

\begin{abstract}
In business-to-business relations, contracts serve both as a protection mechanism of trading partners, as well as a prescription document for activities to be executed by the parties. The processes of contract establishment and its enactment are often expensive and time consuming. E-contracting aims at automation of these processes, making them faster and cheaper. For the design of an information system for support of e-contracting, a clear vision of the e-contracting processes is required. In this paper, we introduce a process model for flexible business-to-business e-contracting. To separate concerns, we distinguish structured function and communication perspectives of $e$ contracting processes complemented with consistency rules. The proposed approach allows achieving completeness and consistency in building complex contracting processes.
\end{abstract}

\section{INTRODUCTION}

Business process modeling and reengineering aims at improving the efficiency and effectiveness of business processes that are executed in a company. Initially, only intra-organizational business processes were considered. Software applications with different levels of complexity are provided for the coordination and automation of intra-organizational processes. After the rapid development of information technology, the possibility for the support of cross-organizational business processes emerged (Grefen, 2000), (Alonso, 1999), (CrossFlow, 2000). Many research institutes and standardization efforts, e.g. ebXML (ebXML, 2001), RosettaNet (RosettaNet, 2001), work on the problem of modeling cross-organizational processes and realization of supporting information systems. Business-to-business contracting is a key example that faces this problem. It governs most business transactions and it comprises a collection of coherent intra- and cross-organizational activities. The choice of the activities to be performed during contracting and the order of execution is context dependent. This adds for the complexity of the process.

Standard paper contracting processes are often slow and require involvement of human actors in all contracting phases. Electronic contracting provides faster and cheaper contract establishment and offers new opportunities to the partners, e.g., 
micro-contracting (Grefen, 2002). However, implementation of information technology for the support of business-to-business electronic contracting requires a clear vision of the activities that are to be performed by the participating companies. In this paper, we present an approach to achieve this.

We describe a method for process modeling of flexible business-to-business econtracting. The modeling approach is based on two perspectives of the econtracting process, i.e., function and communication perspectives. We model the cross-organization activities in a separate view, in order to achieve coherence of communication between parties. Use of process decomposition and process inheritance in the function and communication views respectively, allows achieving a structured and complete model. The two perspectives do not suffice for composing e-contracting processes, as they do not specify sequence, mutual exclusion, etc. of activities. For this reason, we define a set of consistency rules that are used for the specification of the relations between the e-contracting activities. The relation specification facilitates the construction of concrete e-contracting processes.

The combination of function and communication perspectives and the consistency rules provides a tool for the construction of complete and correct concrete e-contracting process specifications. The model can be used for analysis of existing e-contracting process specifications as well. Requirements that are not satisfied or inconsistencies in existing specifications can be discovered. A software architecture is required for realization of e-contracting systems. The elaboration of this software architecture demands clear description of the e-contracting process. The model proposed in this paper is the basis for obtaining requirements for an econtracting system.

This paper is organised as follows. In Section 2, the approach for the econtracting process model is described. In Sections 3 and 4, the function and communication perspectives of the model are depicted. Consistency rules that are applied to processes are explained in Section 5. To show the use of the defined model, we use it in Section 6 to construct sample concrete processes. Finally, we draw conclusions on this paper and outline future research issues.

\section{MODELLING APPROACH}

In this section, we describe our modeling approach and present related work in this field. To provide a complete and consistent model of the e-contracting process, we separate concerns by elaborating different perspectives of the process. To achieve completeness, we depict a function perspective of e-contracting activities. In this view, e-contracting activities are specialized at several levels of abstraction. This perspective provides a complete picture of e-contracting activities to a certain level of specialization. Its hierarchical presentation allows further specialization for the support of specific context requirements. Specific issues of the business domains (such as the insurance domain) can be addressed in this way.

E-contracting is a blend of intra- and cross-organizational activities. An econtracting model should guarantee coherence of cross-organizational activities as well as coherence between the cross- and intra-organizational activities. To achieve this, next to the function perspective we elaborate a communication perspective. The communication perspective is a specialization tree of the different communication 
activity types that occur during e-contracting. The communication hierarchy is coupled with internal processes that are associated with the communication activities. This supports modeling of coherent cross- and intra- organizational process. Being a hierarchy, the communication perspective can be further specialized, if specific business situations require this. The leaves in the communication perspective are a subset of the activity leaves in the function perspective. The communication perspective aims at facilitating the process of defining consistent communication activities in the function perspective. It is applied for the construction of the third level of specialization of the function perspective, where concrete communication activities are identified

To model the relations between the e-contracting activities, we define a set of consistency rules. These rules support the proper construction of the e-contracting process from the identified activities. They can be applied at the different levels of abstraction of the function perspective. For some of the rule types, rule inheritance is applicable.

To position our approach, we relate it to other developments in this field. First, we relate our approach to a research project that was carried out at the University of St. Gallen. A number of efforts exist for the standardization of cross-organizational activities (Angelov, 2001b). For this reason, next, we relate our approach to RosettaNet, which is an established standardization effort for cross-organizational activities.

In (Gisler, 2000), three views on the e-contracting process are depicted. The view on e-contracting activities is equivalent to our highest level of abstraction of the function view. However, the authors describe only briefly the phases and the processes that constitute them. In their paper, a document and a legal perspective are aligned to the activities view. The document view shows the documents delivered at the end of each phase. This is not enough to give a clear vision over the communication activities performed by the parties, through which these documents are delivered. In the legal perspective, legal issues are discussed that are not of importance to our approach. The three views are described at a high level of abstraction and only sketch the general characteristics of the e-contracting process. In our work, we describe in greater details the contracting process, reaching level of concrete contracting activities. As already explained, to achieve coherence of communication activities between parties, we distinguish a separate communication view in the model.

RosettaNet is a standardization effort aiming at a description of the crossorganizational business processes. In RosettaNet, a three level hierarchy of the activities of the collaborating parties is used to guarantee completeness of the standard. This hierarchy is built as a specialization of the domain of e-business supply chain activities. In RosettaNet, in contrast to our approach, no attention is paid to the internal business processes and their relation to the cross-organizational processes. As a result, only the e-contracting communication activities can be extracted from this standard. In this paper, we look towards description of the complete e-contracting process. 


\section{FUNCTION PERSPECTIVE}

In this section, the function perspective of the e-contracting process model is described. The perspective is presented as a combination of a subtyping hierarchy and collection relations. We distinguish three levels of abstraction in the hierarchy. Each level is briefly discussed below.

An e-contracting process consists of a number of e-contracting phases. A phase constitutes of activities, specific for a stage of the e-contracting process. These phases are successively executed in the time. We distinguish four phases: information, pre-contractual, contracting and enactment phases (see Figure 1-phase level). In the information phase, general preparations are made, information is provided (for a request or offer of services) and possible partners are identified. In the pre-contracting phase, preparatory contracting processes are performed. In the contracting phase, the contract is negotiated and established. During the enactment phase, the contract is executed and accompanying activities are performed. For a successful e-contracting process, at least the last two phases must take place (Angelov, 2002a) (this requirement can be defined using the consistency rules described in Section 5).

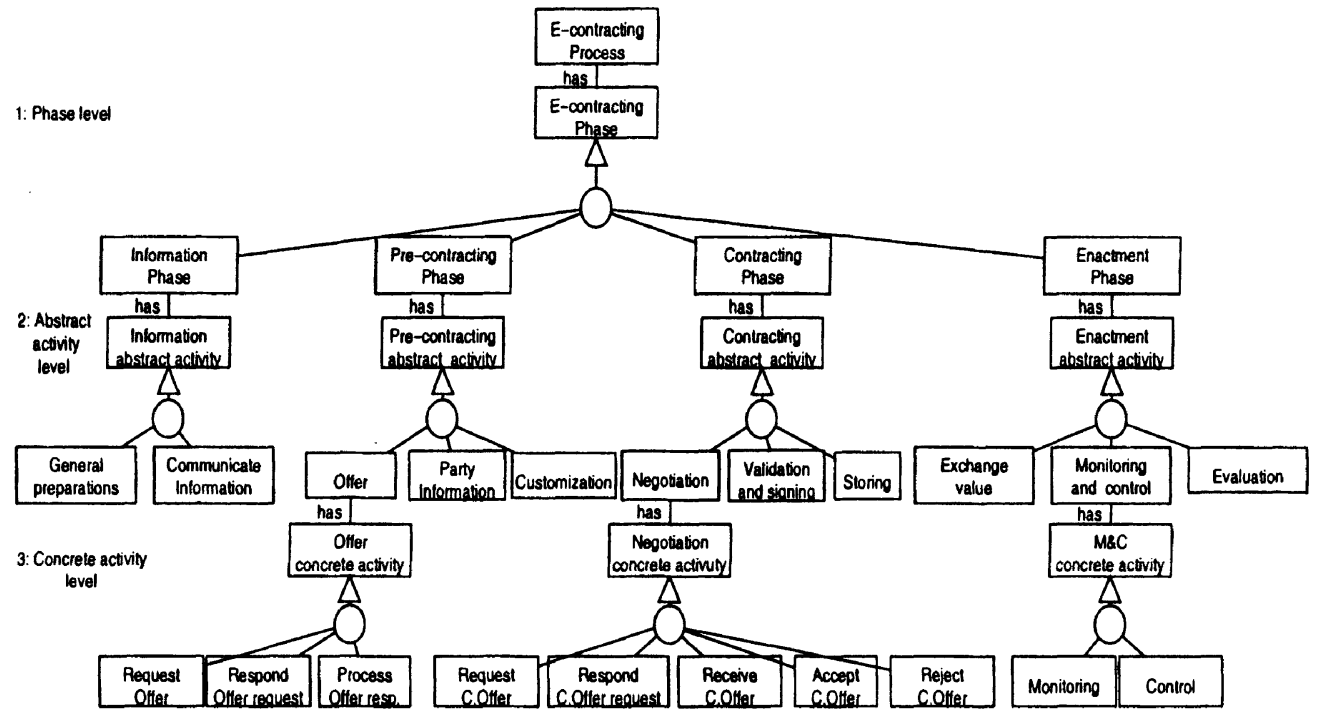

Figure 1 - Function perspective

Each phase contains abstract activities. Abstract activities for each phase are sub-typed to specific abstract activities. They form the second level of abstraction. Abstract activities are a collection of concrete activities that form a logical unit and that in combination deliver a value to the parties. For example, the offer activity is a collection of sub-activities that support the exchange of offers between parties (see concrete activity level). Concrete activities form the third level of abstraction of the function perspective. Figure 1 shows a sample specialization of the offer, negotiation, and monitoring and control concrete activities. For reasons of brevity, the other specializations are not depicted. A detailed description is available in (Angelov, 2002b).

The activities identified at the concrete activity level are leaves in the function perspective. This level of specialization is sufficient for modeling standard e- 
contracting processes. Depending on the business situation, specific activities that are performed during e-contracting might be required. These specific activities can be specialized from the leaf activities in the function perspective. In this way the defined activity tree can be specialized to new levels of detail, e.g., domain level, company level, service level, etc.

In the sequel of the paper, we concentrate on the offer abstract activity. The offer abstract activity is best used to illustrate the benefits of the proposed approach. It contains the request offer, respond offer request, and process offer response concrete activities.

\section{COMMUNICATION PERSPECTIVE}

The function perspective is required to guarantee completeness of the model. At the abstract activity level, the level of specialization allows to distinguish activities as intra- or cross-organizational. Internal activities can vary in their concrete specification, depending on the parties and the business context. However, wellformed communication for the cross-organizational activities, i.e., communication activities between parties, is required to guarantee coherence of the communication activities of parties and thus to guarantee successful exchange of information between parties. Additionally, coherence between the communication activities and the internal activities associated to them must be guaranteed. To specialize crossorganizational activities in the function view and to guarantee their consistency, we need a communication perspective.

In this section, we discuss the communication perspective of the e-contracting process (see Figure 2).

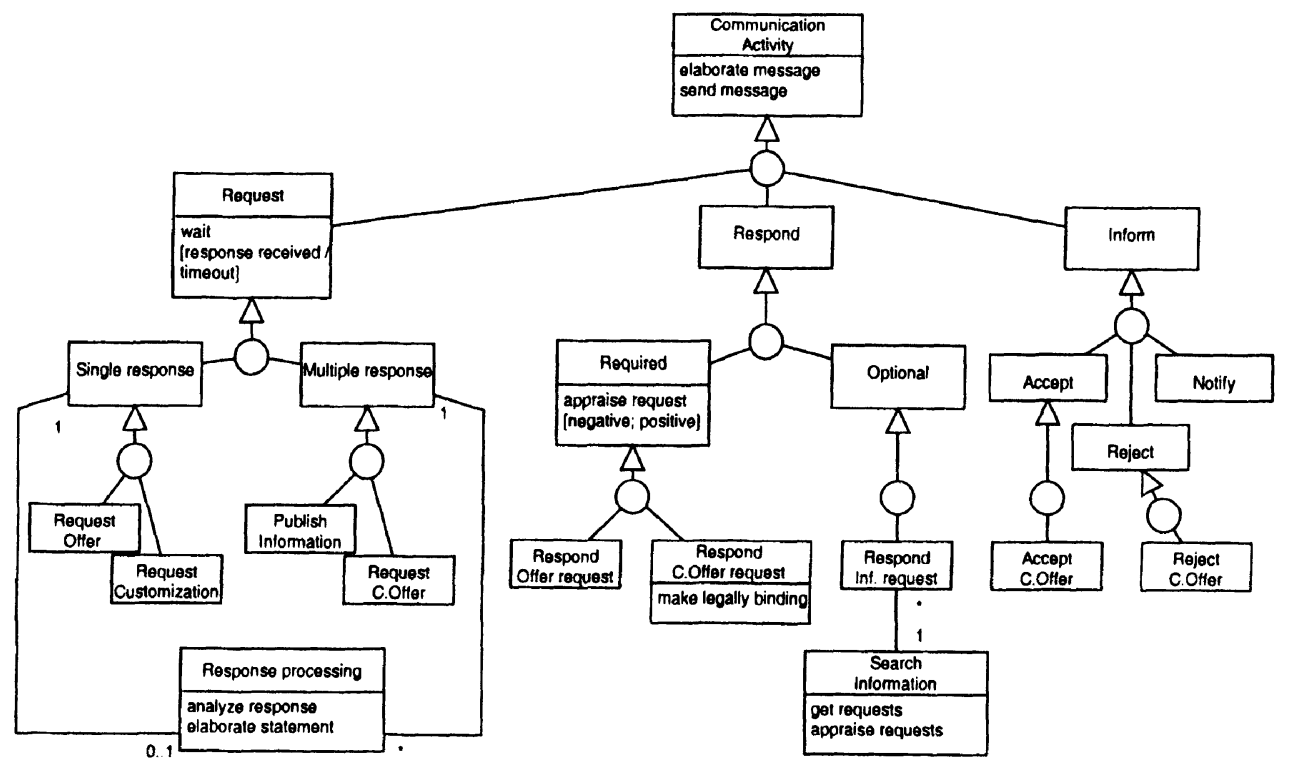

Figure 2 - Communication perspective 
Communication processes are sub-typed from a root communication activity. We specialize the communication activity into request, respond and inform activities. This set of activity types covers all aspects of a communication process. The defined communication activity tree, with this level of specialization, suffices to represent the cross-organizational perspective. It defines the communication patterns for the communication activities and thus it guarantees their consistency. All concrete communication activities can be specialized from this last level. However, to achieve coherence between the intra- and cross-organizational activities, we add to this perspective the internal activities that are directly connected to the communication activities. Such activities are, for example, response processing (i.e. when a party receives a response from another party resulting from previous request activity) and search activities (i.e. when a party searches in a market place to find requests for information from other parties). By adding intra-organizational activities in this view, communication activities and internal activities associated to them are linked to each other. In Figure 2, the internal activities are positioned below the communication hierarchy. Internal activities cause a third level of specialization of communication activities to be defined. We specialize the communication activities according to their relation with the internal activities. A request activity is specialized into requests that expect a single response and requests that expect multiple responses. The internal activity according to which they are specialized is the response processing activity. For example, the request for offer expects only one response, while a request for information, published in a market place, expects many responses from different parties. Analogously, the respond activity is specialized to a required and optional response. Only the response to a request for information (published in a market place) is optional. For all other response activities, the requesting party requires response. For reasons of brevity, only several leaf activities are given in Figure 2 as examples for specialization from the third level. Using the defined communication perspective, we sub-type the crossorganizational abstract activities in the function perspective, e.g., the offer abstract activity.

To facilitate the construction of concrete communication activities, we exploit another feature of the constructed hierarchy, i.e., the inheritance of activities. Communication activities consist of tasks. For example, every communication activity consists of at least two tasks, i.e., elaborate message and send message. We use the communication hierarchy for inheritance of tasks to sub-activities. For example, the request activity inherits elaborate request and send request tasks from the root communication activity. We add a wait state and a triggering event (response received or timeout occurs) for the request activity, as each request waits for response. This inheritance of tasks throughout the tree requires adding only activity specific tasks to the leaf activities and facilitates construction of concrete activities, as we show in Section 6.

\section{CONSISTENCY RULES}

In Sections 3 and 4, we have presented two perspectives of the e-contracting process. These two perspectives do not contain information about the temporal and existence relationships between the executed activities. To provide information 
about these relations, an appropriate notation is required. In this section, a notation that is used to represent this information is described. We define a set of consistency rules that are used to model the activity constraints. A textual notation is used for their definition. There are also graphical notations to represent rules for temporal precedence of activities that can be used as well, e.g. (Jackson, 1997). We define the following relations:

$\operatorname{SEQUENCE}\left(\mathrm{A}_{1}, \mathrm{~A}_{2}\right)$ : The relation expresses that the execution of $A_{1}$ has to precede that of $\mathrm{A}_{2}$. For example, SEQUENCE(Offer, Negotiation) shows that the offer exchange activity precedes the negotiation activity.

EXISTENCE $\left(\mathbf{A}_{1}, \mathbf{A}_{2}\right)$ : The SEQUENCE relation can be strengthened by the EXISTENCE $\left(A_{1}, A_{2}\right)$ relation, which expresses the requirement that activity $A_{2}$ can be executed only when the activity $A_{1}$ has been executed. An example would be EXISTENCE(Offer, Customization). A customization activity can be executed only when an offer has been exchanged between the parties.

$\operatorname{EXCLUDE}\left(\mathbf{A}_{1}, \mathbf{A}_{\mathbf{2}}\right)$ : This relation shows that both activities are mutually exclusive, e.g., EXCLUDE(Accept contract offer, Reject contract offer).

REQUIRED( $\left.\mathbf{A}_{\mathbf{1}}\right)$ : The REQUIRED operator indicates that the activity given as an argument must be executed and is not optional, e.g. REQUIRED(Exchange value).

This set can be extended with other rules, if additional constraints must be imposed.

The hierarchical representation of the function perspective allows rule inheritance for the SEQUENCE and EXCLUDE operators to be applied. Defining SEQUENCE(Contracting phase, Enactment phase), means that all sub-activities of the two phases inherit the defined rule. This rule inheritance is not valid for the EXISTENCE and REQUIRED operators.

\section{E-CONTRACTING PROCESS DESIGN}

The described model in combination with the defined consistency rules does not define a unique e-contracting process. Business-to-business e-contracting varies in the performed activities depending on the companies and the business context (Angelov, 2001a). The model gives flexibility to construct various concrete econtracting processes, depending on the business context. By applying consistency rules, a company can define different relations between activities, achieving a correct flexible e-contracting process specification.

In this section, the model is used to construct a fragment of a concrete econtracting process specification. Activity diagrams are used as a modeling technique (Eriksson, 2000). In the following example, we concentrate on the construction of cross-organizational activities, as they show the application of both perspectives of the model. Concrete specifications for activities that are entirely internal can vary significantly, depending on the specific company. To construct a 
complete e-contracting process specification, a party starts with identification of the activity leaves that will participate in the process definition. This step might require additional specializations of the activity leaves from the functional perspective. Next, a company uses the consistency rules to define the relations between the identified activities. Then, a process specification for each activity is elaborated.

To demonstrate the use of the defined model, concrete activity diagrams for the offer abstract activity are constructed. As the offer activity is a cross-organizational activity, we use both the function and the communication view for the specification construction. First, we identify the activity in the function activity tree. The activity is a sub-activity of the pre-contractual phase. Next step is to select the activity leaves of the offer activity.

The offer activity is a request-response activity. It does not involve the third communication category, i.e., inform activity (see Figure 2). Next, the communication perspective is used to identify the leaf activities of the offer abstract activity. Usually, the offer activity will start with a request for offer from the consumer in order to get the specifications of the provided service by the supplier. As each offer request is directed to one company, a single response by the supplier is required in return. In this way, the request offer and respond offer request activities are identified in the request and respond branches respectively of the communication perspective. When received, the response (i.e., the offer) has to be processed by the consumer. As a result, three activity leaves that specialize the offer activity are found, i.e., request offer, respond offer request and process offer response activities (we use for each of them the abbreviations $\mathrm{RqO}, \mathrm{RpORq}$, PrORp respectively, and $A_{O}$ to denote the set of all three activities). This example shows that the communication perspective facilitates easy definition of communication activities. In the rest of the example, we assume that a company has already identified all the leaf activities that build its e-contracting process.

Next step is to define consistency rules on each of the three activities. We start with using the SEQUENCE operator:

1. We have:

SEQUENCE (Information phase, Pre-contracting phase), defined in the function perspective.

From this we infer:

SEQUENCE (Information phase, $A_{0}$ )

Additionally we define:

SEQUENCE (RqO, RpORq), and

SEQUENCE (RpORq, PrORp).

2. To strengthen the SEQUENCE operations, we use the EXISTENCE operator:

EXISTENCE (RpORq, PrORp)

EXISTENCE (General preparations, RpORq), indicates that general provisions must be available in order to be attached to the offer when the response is elaborated.

3. Finally, the REQUIRED operator gives:

REQUIRED (RPORq) and

REQUIRED (PrORp) 
Before specifying concrete processes, a start event that triggers the activity and if necessary an end event for it must be defined. In our example, an internal for the company event offer needed will trigger the RqO activity. The event offer request, generated by the $\mathrm{RqO}$ activity or by internal event at the supplier side, will trigger the RpORq activity. Finally, the event offer received at the consumer side will trigger the PrORp activity.

Next, activity diagrams can be constructed. First, we construct the request offer specification. Using the inheritance of tasks, described in the communication perspective, we see that the offer request contains three basic activities, i.e., elaborate offer request, send offer request, and wait state (see Figure 3).

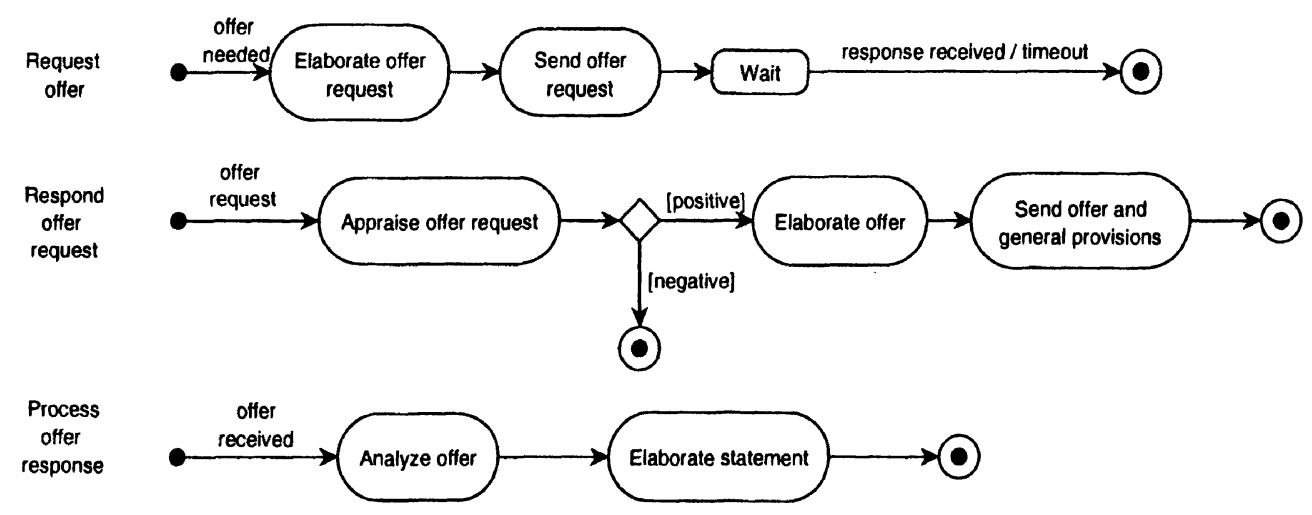

Figure 3 - Offer activity specification

Analogously, using the communication perspective, the respond offer request and process offer response activity diagrams are constructed. The responding company receives the request for offer and appraises the request. If the request for offer is approved (a positive decision is taken to answer to the request), the company elaborates an offer and sends it. The elaborate message and send message activities are inherited from the root communication activity in the communication perspective and are applied for the respond offer activity context. The offer is sent together with the general provisions prepared during the general preparations activity. The response is received by the requesting party and is analyzed. Based on the analyses, a statement on the received offer is elaborated.

The described specification of concrete activities is based solely on the function and communication perspectives. The example demonstrates that the two perspectives and the consistency rules are a powerful tool that allows consistent and complete e-contracting process specifications to be achieved.

\section{CONCLUSION AND FUTURE WORK}

In this paper, we have described an e-contracting process model. The model is based on two perspectives, i.e., function and communication. These perspectives, in combination with a set of consistency rules provide for tools to guard completeness and consistency of e-contracting processes. Based on the two perspectives and the set of consistency rules, we define an approach for specification of concrete e- 
contracting process. The model can be used for the analysis of existing e-contracting process specifications as well. To illustrate the advantages of the proposed modeling approach, the model is used to construct a fragment of a concrete e-contracting process specification.

An information system is required for the partial or full automation of the econtracting process. However, detailed reference architecture for the support of econtracting processes does not yet exist. This research work is part of the econtracting system environment analysis. A data model is required in addition to the process model. More specifically, the e-contract content and its representation are to be researched. The process and data models together allow collecting requirements for an information system that supports an e-contracting process in its four phases. This preliminary work is a step towards the construction of detailed e-contracting reference architecture, which is our research goal.

\section{REFERENCES}

1. Alonso G, Fiedler U, Hagen C, Lazcano A, Schuldt H, Weiler N. WISE: business to business ecommerce. Procs. 9th International Workshop on Research Issues on Data Engineering: Information Technology for Virtual Enterprises. Australia. 1999.

2. Angelov S, Grefen P. A conceptual framework for B2B electronic contracting. Procs. 3rd IFIP Working Conference on Infrastructures for Virtual Enterprises. Portugal. 2002 (a).

3. Angelov S, Grefen P. An approach for flexible B2B e-contracting process modeling. Twente University: CTIT Technical Report. 2002(b). http://www.ctit.utwente.n1/publications/Tr01/.

4. Angelov S, Grefen P. A framework for the analysis of B2B electronic contracting support. Procs. 4th Edispuut Conference - Multidisciplinary Perspectives on Electronic Commerce. 2001(a).

5. Angelov S, Grefen P. B2B eContract handling - a survey of projects, papers and standards. Twente University: CTIT Technical Report 01-21. 2001(b).

6. CrossFlow project. 2000. http://www.crossflow.org/.

7. ebXML. Technical architecture specification v1.0.4. ebXML. 2001. http://www.ebxml.org.

8. Eriksson H.-E, Penker M. Business modeling with UML: business patterns at work. John Wiley \& Sons. New York. 2000.

9. Gisler M, Stanoevska-Slabeva K, Greunz M. Legal aspects of electronic contracts. Infrastructures for Dynamic Business-to-Business Service Outsourcing (IDSO'00). Stockholm. 2000.

10. Grefen P, Angelov S. On $\tau-, \mu-, \pi$-, and $\varepsilon$-Contracting. To appear in Procs. Web Services, e-Business, and the Semantic Web. Canada. 2002.

11. Grefen P, Aberer K, Hoffner Y, Ludwig H. CrossFlow: Cross-organizational workflow management in dynamic virtual enterprises. International Journal of Computer Systems Science \& Engineering, Vol. 15, No. 5, pp. 277-290; 2000.

12. Jackson $\mathbf{M}$, Twaddle $\mathbf{G}$. Business process implementation building workflow systems. AddisonWesley. 1997.

13. RosettaNet. RosettaNet implementation framework: core specification (RNIF 02). RosettaNet. 2001. http://www.rosettanet.org. 